\title{
Hybrid finite elements for the computation of sandwich plates
}

\author{
Vincent Manet, Woo-Suck Han and Alain VAUtrin \\ Ecole des Mines de Saint-Etienne \\ Material and Mechanical Department \\ 158, cours Fauriel \\ 42023 Saint-Etienne cedex 2
}




\begin{abstract}
In this article, finite elements are developed on the basis of the displacements and the Pian and Tong functionals with help of Lagrange multipliers.

These elements represent sandwich structure in an accurate way, especially at interfaces, where the equilibrium state is satisfied.

Developed elements are assessed and compared on several examples of static linear problems.
\end{abstract}

\title{
Notations
}

In this article, Einstein summation convention is used, but does not apply between superior indices, which designate element, and inferior indices, which denotes components: it only applies between inferior indices.

We note $\boldsymbol{T}=\boldsymbol{\sigma} \boldsymbol{n}$ (or $T_{i}=\sigma_{i j} n_{j}$ ) the projection of $\boldsymbol{\sigma}$ on the outgoing normal $\boldsymbol{n}$ to a considered domain $\Omega$ on its boundary $\Gamma$ : it is the normal trace of the stress tensor on $\Gamma$.

$\mathbb{K}$ stands for the stiffness matrix of the whole system, $\mathbb{Q}$ the overall vector of nodal unknowns and $\mathbb{F}$ the overall vector of equivalent forces.

\section{Introduction}

The sandwich plate have become an important structural component for industrial applications, especially for the transport field: aeronautics, automobile, railroads, shipbuilding... The increasing interest for this component in the structural design led us to develop powerful analysis tools by FEM, able to take into account correctly its specificity. At any way, its modelling stands always by a lack of precision on the numerical analysis.

A sandwich structure is a laminated one composed of three layers whose mechanical and geometrical properties are very different:

- the core, thick with low stiffness and density, is essentially subjected to transversal shear effects;

- two skins, thins and with high stiffness, wrap the core and work in plane. 
A typical sandwich structure is shown, in the $2 \mathrm{D}$ case, in figure 1 . Figure 2 gives the same illustration, but from the FEM point of view.

Let us note that the behaviour of sandwich plates is very different from that of laminated plates, particularly when we consider failure modes: rupture, buckling, local denting... ${ }^{1}$.

Whatever chosen materials may be for the core and skins, we retain in the present study the following assumptions at the interfaces between different layers:

- the displacement field is continuous, i.e. the adhesion between layers is perfect;

- the thickness of the adhesive is sufficiently small to be neglected.

Hence, in order to ensure a correct modelling of sandwich plates by FEM, the following conditions must be fulfilled:

- the continuity of the displacement field;

- the force equilibrium state, especially at the interface between layers.

Transverse shear effects must be taken into account: this point is extremly important since the core is essentially subjected to shear effects. The plane stress assumption, often used in the classical laminate theory, must not be employed since the core can be compressed through the thickness in a significant way due to its flexibility.

In the design process, stresses at interfaces, and particularly interlaminar stresses, play a preponderant role. For example, transverse shear stresses, which are much smaller than other stress components, can overhang all other phenomena $a^{2,3,4,5}$. They play also a crucial role in failure modes due to their particular influence ${ }^{1,6}$.

The present work concerns the development of hybrid sandwich elements taking the aforementioned points into account, i.e. representing the behaviour of the sandwich plate in a more realistic way and respecting physical phenomena at the interfaces between the skins and the core.

These elements are principally based on hybrid formulation introducing Lagrange multipliers at the interfaces. The advantages of this formulation are the following: 
- it can verify simultaneously the continuity of displaments and the force equilibrium state at the interfaces with a minimum degrees of freedom (DOF) by element;

- it can be developed in a systematical way and so be easily implemented in existing FEM codes, which is not always the case with elements based on Reissner's mixed formulation with relaxation of redundant $\mathrm{DOF}^{7}$.

\section{FE Modelling}

With displacement elements, which only use the displacement field as unknown, stresses are obtained by numerical derivation which induces numerical errors. In this case, the force equilibrium state between elements, especially at interfaces, cannot be fulfilled.

Mixed methods, based on Reissner's functional for example, take independently displacement field and stress field as variables. So both fields are completely continuous between elements. But, the normal stress component tangential to the interface is not obliged to be continuous. In other word the continuity of this component is excessive. An other drawback is that the number of unknowns by element rapidly increases.

Hybrid mixed methods can also take these two fields as variables, but not in the same manner: one field is generally assumed in the element, an other one on its boundary. The functional generally associated with the adjective hybrid is that proposed by Pian and Tong ${ }^{8}$. This functional assumes the stress field in the element and the displacement field on its boundary. So long that all nodal variables (displacements and stresses) are used, both fields are continuous between elements. The stress field being only interpolated within the element, it can be "condensed", i.e. removed from the nodal variables, so that only displacements appear as nodal variables. But, when the stress field is condensed, the continuity of this field between elements cannot be ensured. The condensed Pian and Tong's functional has a form equivalent to the displacement functional, but yields a relation between stresses and displacements which does not contain any derivative.

A way of building hybrid elements is to use Lagrange multipliers on the interface to join subdomains. These multipliers are then physically 
interpreted. The main advantage of this method is its flexibility of use since only chosen conditions are imposed and only where it is required. It is why we use this method to build finite elements able to describe the behaviour of sandwich plates.

In the following, we note $\boldsymbol{N}_{\boldsymbol{u}}$ the matrix of the shape functions for displacements, $\boldsymbol{N}_{\boldsymbol{\sigma}}$ for the stress field if it appears at nodal variable, and $\boldsymbol{N}_{\boldsymbol{\lambda}}$ for Lagrange multipliers.

These functions can be as wanted in each sub-element; nevertheless we use quadratic formulations in all directions of the space (dimension 2 or 3 ) for displacements and stresses. The matrix $\boldsymbol{N}_{\boldsymbol{\lambda}}$, as for it, is quadratic too, but along interfaces (dimension 1 or 2 ).

\subsection{Element 1: minimum of total potential en- ergy and Lagrange multipliers}

In order to verify that Lagrange multipliers are able to fulfill our requirements, we developed the first element in a very simple way: each layer being modelled by a subdomain in displacements, Lagrange multipliers are introduced at the interfaces.

The total potential energy for the layer $i$ is written by:

$$
\Pi^{i}=\int_{\Omega^{i}}{ }^{\frac{1}{2}} \boldsymbol{\varepsilon}^{i} \boldsymbol{\sigma}^{i}-{ }^{t} \boldsymbol{U}^{i} \overline{\boldsymbol{f}}_{\boldsymbol{\Omega}}^{i} \mathrm{~d} \Omega-\int_{\Gamma_{\sigma}^{i}}{ }^{t} \boldsymbol{U}^{i} \overline{\boldsymbol{T}}^{i} \mathrm{~d} \Gamma \quad \forall i \in\{1,2,3\}
$$

where the exponent $i$ indicates the layer $i,{ }^{t} \varepsilon$ the transpose of the strain vector, $\boldsymbol{\sigma}$ the stress vector, ${ }^{\boldsymbol{U}} \boldsymbol{U}$ the transpose of the displacement vector, $\overline{\boldsymbol{f}}_{\Omega}$ the body force vector, $\Omega$ a volume, $\overline{\boldsymbol{T}}$ the vector of prescribed surface forces applied on $\Gamma_{\sigma}$ which is a part of the boundary of $\Omega, \Gamma_{u}$ being the rest of the boundary where displacements are prescribed.

At each interface, we introduce the displacement continuity conditions using Lagrange multipliers $\boldsymbol{\lambda}$ :

$$
\Pi_{I}^{i}=\int_{\Gamma_{I}^{i}}{ }^{t} \boldsymbol{\lambda}^{i}\left(\boldsymbol{U}^{i}-\boldsymbol{U}^{i+1}\right) \mathrm{d} \Gamma \quad \forall i \in\{1,2\}
$$

where $\Gamma_{I}^{i}$ is the interface between layers $i$ and $i+1$, and ${ }^{t} \boldsymbol{\lambda}$ the transpose of Lagrange multiplier vector. 
The total potential energy for the first hybrid sandwich element is:

$$
\Pi=\sum_{i=1}^{3} \Pi^{i}+\sum_{i=1}^{2} \Pi_{I}^{i}
$$

and its virtual variation:

$$
\begin{aligned}
\delta \Pi= & \sum_{i=1}^{3}\left(\int_{\Omega^{i}} \delta^{t} \varepsilon^{i} \boldsymbol{D}^{i} \varepsilon^{i}-\delta^{t} \boldsymbol{U}^{i} \overline{\boldsymbol{f}}_{\boldsymbol{\Omega}}^{i} \mathrm{~d} \Omega-\int_{\Gamma_{\sigma}^{i}} \delta^{t} \boldsymbol{U}^{i} \overline{\boldsymbol{T}}^{i} \mathrm{~d} \Gamma\right) \\
+ & \sum_{i=1}^{2}\left(\int_{\Gamma_{I}^{i}} \delta^{t} \boldsymbol{U}^{i}\left(\boldsymbol{T}^{i}-\boldsymbol{\lambda}^{i}\right)+\delta^{t} \boldsymbol{U}^{i+1}\left(\boldsymbol{T}^{i+1}+\boldsymbol{\lambda}^{i}\right) \mathrm{d} \Gamma\right. \\
& \left.-\int_{\Gamma_{I}^{i}} \delta^{t} \boldsymbol{\lambda}^{i}\left(\boldsymbol{U}^{i}-\boldsymbol{U}^{i+1}\right) \mathrm{d} \Gamma\right)
\end{aligned}
$$

with the generalized Hooke's matrix $\boldsymbol{D}$ which relates stresses to strains such as $\boldsymbol{\sigma}=\boldsymbol{D} \varepsilon$.

At this point, only the continuity of the displacement field seems to be ensured. Let us identify the Lagrange multipliers: the stationarity of our functional yields the following equilibrium equations along the interfaces $\Gamma_{I}^{i}$ :

$$
\left\{\begin{array}{rl}
\lambda_{j}^{i} & =T_{j}^{i}=-T_{j}^{i+1} \\
U_{j}^{i} & =U_{j}^{i+1}
\end{array} \quad \forall i \in\{1,2\}, \forall j \in\{1,2,3\}\right.
$$

Hence, along interfaces, Lagrange multipliers are equal to the stress components we wanted (interlaminar stresses) and the equilibrium state is fulfilled:

$$
T_{j}^{i}+T_{j}^{i+1}=0 \quad \forall i \in\{1,2\}, \forall j \in\{1,2,3\}
$$

We verify that the defined functional, even if based on a displacements formulation, has displacements and the interlaminar stress components as nodal unknowns. All required conditions are fulfilled by the model.

The discretization of equation (2) leads to the matrix system:

$$
\mathbb{K} \mathbb{Q}=\mathbb{F}
$$


with:

$$
\begin{gathered}
\mathbb{K}=\left[\begin{array}{ccc|cc}
K_{1}+H_{11} & \mathbb{O} & \mathbb{O} & Q_{11} & \mathbb{O} \\
\mathbb{O} & K_{2}+H_{21}+H_{12} & \mathbb{O} & Q_{21} & Q_{22} \\
\mathbb{O} & \mathbb{O} & K_{3}+H_{32} & \mathbb{O} & Q_{32} \\
\hline{ }^{t} Q_{11} & { }^{t} Q_{21} & \mathbb{O} & \mathbb{O} & \mathbb{O} \\
\mathbb{O} & { }^{t} Q_{22} & { }^{t} Q_{32} & \mathbb{O} & \mathbb{O}
\end{array}\right] \\
\mathbb{Q}=\left\{\begin{array}{l}
q^{1} \\
q^{2} \\
q^{3} \\
L^{1} \\
L^{2}
\end{array}\right\}
\end{gathered}
$$

and:

$$
\boldsymbol{K}_{\boldsymbol{i}}=+\int_{\Omega^{i}}{ }^{t}\left(\mathcal{L} \boldsymbol{N}_{\boldsymbol{u}^{i}}\right) \boldsymbol{D}^{i}\left(\mathcal{L} \boldsymbol{N}_{\boldsymbol{u}^{i}}\right) \mathrm{d} \Omega
$$

where $\mathcal{L}$ is the matrix of the differential operator relating strains to displacements $(\varepsilon=\mathcal{L} U)$.

$$
\begin{gathered}
\boldsymbol{f}_{\boldsymbol{u}^{i}}=+\int_{\Omega^{i}}{ }^{t} \boldsymbol{N}_{\boldsymbol{u}^{i}} \overline{\boldsymbol{f}}_{\boldsymbol{\Omega}}{ }^{i} \mathrm{~d} \Omega+\int_{\Gamma_{\sigma}^{i}}{ }^{t} \boldsymbol{N}_{\boldsymbol{u}^{i}} \overline{\boldsymbol{T}}^{i} \mathrm{~d} \Gamma \\
\boldsymbol{Q}_{\boldsymbol{i j}}=(-1)^{2-\delta_{i j}} \int_{\Gamma_{I}^{j}}{ }^{t} \boldsymbol{N}_{\boldsymbol{u}^{i}} \boldsymbol{N}_{\boldsymbol{\lambda}^{j}} \mathrm{~d} \Gamma \\
\boldsymbol{H}_{\boldsymbol{i j}}=+\int_{\Gamma_{I}^{j}}{ }^{t} \boldsymbol{N}_{\boldsymbol{u}^{i}} \boldsymbol{M}^{i} \boldsymbol{D}^{i} \mathcal{L} \boldsymbol{N}_{\boldsymbol{u}^{i}} \mathrm{~d} \Gamma
\end{gathered}
$$

where $\boldsymbol{M}^{i}$ is the matrix of cosines of the outgoing normal of $\Omega^{i}$ on $\Gamma^{i}$ with components $n_{i}$ :

$$
\boldsymbol{M}^{i}=\left[\begin{array}{ccc}
n_{1} & 0 & n_{2} \\
0 & n_{2} & n_{1}
\end{array}\right]
$$

in the $2 \mathrm{D}$ case, and

$$
\boldsymbol{M}^{i}=\left[\begin{array}{cccccc}
n_{1} & 0 & 0 & 0 & n_{3} & n_{2} \\
0 & n_{2} & 0 & n_{3} & 0 & n_{1} \\
0 & 0 & n_{3} & n_{2} & n_{1} & 0
\end{array}\right]
$$

in the $3 \mathrm{D}$ space.

Equations (5) and (6) are identical to the ones of the displacements method and ensure the internal equilibrium of each sub-element. 
Equation (7) leads to the continuity of the displacement field at interfaces, and equation (8) permits to evaluate the Lagrange multipliers and hence to reach the equilibrium state of stresses at interfaces.

The advantage of this formulation is the same as with all Lagrange multipliers method: each layer is modelled independently from the others, the model is also easy to build.

The main disadvantage is that the $\boldsymbol{M}^{i}$ matrices being non-symmetrical (they are not even square), it is the same for $\boldsymbol{H}_{\boldsymbol{i} \boldsymbol{j}}$, and finally for the stiffness matrix $\mathbb{K}$.

The matrix $\mathbb{K}$ is not symmetrical nor definite-positive.

\subsection{Element 2: mixed hybrid formulation}

An other possible approach is to use a multi-field functional, in our case the displacement and stress fields, in order to model each subdomain.

For that, we propose to use the Pian and Tong hybrid mixed formulation for each layer $i$ :

$\Pi^{i}=-\frac{1}{2} \int_{\Omega^{i}}{ }^{t} \boldsymbol{\sigma}^{i} \boldsymbol{S}^{i} \boldsymbol{\sigma}^{i} \mathrm{~d} \Omega+\int_{\Gamma}{ }^{t} \boldsymbol{T}^{i} \boldsymbol{U}^{i} \mathrm{~d} \Gamma-\int_{\Gamma_{\sigma}}{ }^{t} \overline{\boldsymbol{T}}^{i} \boldsymbol{U}^{i} \mathrm{~d} \Gamma \quad \forall i \in\{1,2,3\}$

with $\boldsymbol{S}$ is the compliance matrix $\left(\boldsymbol{S}=\boldsymbol{D}^{-1}\right)$.

Nodal unknowns being displacements and stresses, it is not necessary to impose any continuity condition at interfaces.

The functional of this hybrid sandwich element is:

$$
\Pi=\sum_{i=1}^{3} \Pi^{i}
$$

and its variation:

$$
\begin{aligned}
\delta \Pi=\sum_{i=1}^{3} & \left(-\int_{\Omega} \delta^{t} \boldsymbol{\sigma}^{i} \boldsymbol{S}^{i} \boldsymbol{\sigma}^{i} \mathrm{~d} \Omega+\int_{\Gamma} \delta^{t} \boldsymbol{T}^{i} \boldsymbol{U}^{i} \mathrm{~d} \Gamma\right. \\
& \left.+\int_{\Gamma} \delta^{t} \boldsymbol{U}^{i} \boldsymbol{T}^{i} \mathrm{~d} \Gamma-\int_{\Gamma_{\sigma}} \delta^{t} \boldsymbol{U}^{i} \overline{\boldsymbol{T}}^{i} \mathrm{~d} \Gamma\right)
\end{aligned}
$$

The stationarity of this functional yields to the following equilibrium equations along interfaces $\Gamma_{I}^{i}$ :

$$
\left\{\begin{array}{l}
\sigma_{k l}^{i}=\sigma_{k l}^{i+1} \\
U_{k}^{i}=U_{k}^{i+1}
\end{array} \quad \forall i \in\{1,2\}, \forall k, l \in\{1,2,3\}\right.
$$


We verify that the defined functional leads to the continuity of all components of the stress field, which is excessive, but characteristic of this method.

The discretisation of equation (11) yields the matrix system:

$$
\mathbb{K} \mathbb{Q}=\mathbb{F}
$$

with:

$$
\begin{aligned}
& {\left[\begin{array}{ccc|ccc} 
& & B_{1} & \mathbb{O} & \mathbb{O} \\
& \mathbb{O} & & \mathbb{O} & B_{2} & \mathbb{O} \\
& & & \mathbb{O} & \mathbb{O} & B_{3} \\
\hline{ }^{t} B_{1} & \mathbb{O} & \mathbb{O} & -A_{1} & \mathbb{O} & \mathbb{O} \\
\mathbb{O} & { }^{t} B_{2} & \mathbb{O} & \mathbb{O} & -\boldsymbol{A}_{2} & \mathbb{O} \\
\mathbb{O} & \mathbb{O} & { }^{t} B_{3} & \mathbb{O} & \mathbb{O} & -\boldsymbol{A}_{3}
\end{array}\right] } \\
\mathbb{Q}=\left\{\begin{array}{c}
q^{1} \\
q^{2} \\
q^{3} \\
\tau^{1} \\
\tau^{2} \\
\tau^{3}
\end{array}\right\} & \mathbb{F}=\left\{\begin{array}{c}
f_{u^{1}}^{\prime} \\
f_{u^{2}}^{\prime} \\
f_{u^{3}}^{\prime} \\
\mathbb{O} \\
\mathbb{O} \\
\mathbb{O}
\end{array}\right\}
\end{aligned}
$$

and:

$$
\begin{gathered}
\boldsymbol{A}_{\boldsymbol{i}}=+\int_{\Omega^{i}}{ }^{t} \boldsymbol{N}_{\boldsymbol{\sigma}^{i}} \boldsymbol{S}^{i} \boldsymbol{N}_{\boldsymbol{\sigma}^{i}} \mathrm{~d} \Omega \\
\boldsymbol{f}_{\boldsymbol{u}^{i}}=+\int_{\Gamma_{\sigma}^{i}}{ }^{t} \boldsymbol{N}_{\boldsymbol{u}^{i}} \overline{\boldsymbol{T}}^{i} \mathrm{~d} \Gamma \\
\boldsymbol{B}_{\boldsymbol{i}}=+\int_{\Gamma}{ }^{t} \boldsymbol{N}_{\boldsymbol{u}^{i}} \boldsymbol{M}^{i} \boldsymbol{N}_{\boldsymbol{\sigma}^{i}} \mathrm{~d} \Gamma \\
\boldsymbol{f}_{\boldsymbol{u}^{i}}^{\prime}=+\int_{\Gamma_{\sigma}^{i}}{ }^{t} \boldsymbol{N}_{\boldsymbol{u}^{i}} \overline{\boldsymbol{T}}^{i} \mathrm{~d} \Gamma
\end{gathered}
$$

The symmetrical model directly yields the continuity of all components of displacements and stresses, which is excessive at interfaces.

The matrix $\mathbb{K}$ is symmetrical but not definite-positive. 


\subsection{Element 3: Pian and Tong functional and Lagrange multipliers}

We verified that Pian and Tong model yields an excessive continuity of the stress field, and that the use of Lagrange multipliers with the functional of the total potential energy leads to a non-symmetrical matrix.

We propose to use the Pian and Tong formulation in each subdomain of the sandwich and to re-introduce the displacements continuity through Lagrange multipliers along interfaces.

As for element 1, added conditions for displacements continuity are given by relation (1). The identification of Lagrange multipliers yields, once more, to equation (3). The variational principle of this sandwich hybrid element is the following:

$$
\begin{aligned}
& \delta \Pi=\sum_{i=1}^{3}\left(-\int_{\Omega} \delta^{t} \boldsymbol{\sigma}^{i} \boldsymbol{S}^{i} \boldsymbol{\sigma}^{i} \mathrm{~d} \Omega+\int_{\Gamma} \delta^{t} \boldsymbol{T}^{i} \boldsymbol{U}^{i} \mathrm{~d} \Gamma\right. \\
& \left.+\int_{\Gamma} \delta^{t} \boldsymbol{U}^{i} \boldsymbol{T}^{i} \mathrm{~d} \Gamma-\int_{\Gamma_{\sigma}} \delta^{t} \boldsymbol{U}^{i} \overline{\boldsymbol{T}}^{i} \mathrm{~d} \Gamma\right) \\
& +\sum_{i=1}^{2}\left(\int_{\Gamma_{I}^{i}} \delta^{t} \boldsymbol{U}^{i}\left(\boldsymbol{T}^{i}-\boldsymbol{\lambda}^{i}\right)+\delta^{t} \boldsymbol{U}^{i+1}\left(\boldsymbol{T}^{i+1}+\boldsymbol{\lambda}^{i}\right) \mathrm{d} \Gamma\right. \\
& \left.-\int_{\Gamma_{I}^{i}} \delta^{t} \boldsymbol{\lambda}^{i}\left(\boldsymbol{U}^{i}-\boldsymbol{U}^{i+1}\right) \mathrm{d} \Gamma\right)
\end{aligned}
$$

The discretisation is always of the form:

$$
\mathbb{K} \mathbb{Q}=\mathbb{F}
$$

with, this time:

$$
\mathbb{K}=\left[\begin{array}{ccc|ccc|cc} 
& & & B_{1} & \mathbb{O} & \mathbb{O} & Q_{11} & \mathbb{O} \\
& \mathbb{O} & & \mathbb{O} & \boldsymbol{B}_{2} & \mathbb{O} & \boldsymbol{Q}_{21} & \boldsymbol{Q}_{22} \\
& & & \mathbb{O} & \mathbb{O} & \boldsymbol{B}_{3} & \mathbb{O} & \boldsymbol{Q}_{32} \\
\hline{ }^{t} \boldsymbol{B}_{1} & \mathbb{O} & \mathbb{O} & -\boldsymbol{A}_{1} & \mathbb{O} & \mathbb{O} & \mathbb{O} & \mathbb{O} \\
\mathbb{O} & { }^{t} \boldsymbol{B}_{2} & \mathbb{O} & \mathbb{O} & -\boldsymbol{A}_{2} & \mathbb{O} & \mathbb{O} & \mathbb{O} \\
\mathbb{O} & \mathbb{O} & { }^{t} \boldsymbol{B}_{3} & \mathbb{O} & \mathbb{O} & -\boldsymbol{A}_{3} & \mathbb{O} & \mathbb{O} \\
\hline{ }^{t} \boldsymbol{Q}_{11} & { }^{t} \boldsymbol{Q}_{21} & \mathbb{O} & \mathbb{O} & \mathbb{O} & \mathbb{O} & \mathbb{O} & \mathbb{O} \\
\mathbb{O} & { }^{t} \boldsymbol{Q}_{\mathbf{2 2}} & { }^{t} \boldsymbol{Q}_{\mathbf{3 2}} & \mathbb{O} & \mathbb{O} & \mathbb{O} & \mathbb{O} & \mathbb{O}
\end{array}\right]
$$


and:

$$
\mathbb{Q}=\left\{\begin{array}{c}
q^{1} \\
q^{2} \\
q^{3} \\
\tau^{1} \\
\tau^{2} \\
\tau^{3} \\
L^{1} \\
L^{2}
\end{array}\right\} \quad \mathbb{F}=\left\{\begin{array}{c}
f_{u^{1}}^{\prime} \\
f_{u^{2}}^{\prime} \\
f_{u^{3}}^{\prime} \\
\mathbb{O} \\
\mathbb{O} \\
\mathbb{O} \\
\mathbb{O} \\
\mathbb{O}
\end{array}\right\}
$$

$\boldsymbol{A}_{\boldsymbol{i}}$ matrices are given by relation (13), $\boldsymbol{B}_{\boldsymbol{i}}$ matrices by (15), and $\boldsymbol{Q}_{i j}$ matrices by (7).

Such a system, which is symmetrical, leads to the continuity of all components of displacements and stresses. Lagrange multipliers are redundants.

It is important to notice that $\boldsymbol{A}_{\boldsymbol{i}}$ matrices are invertibles. It is also possible to "condense" the stress field, so that this field will be removed from nodal variables, by expressing it as a function of displacements:

$$
\boldsymbol{\tau}^{i}=-\boldsymbol{A}^{i^{-1} t} \boldsymbol{B} \boldsymbol{q}^{i} \quad \forall i \in\{1,2,3\}
$$

in each layer $i$. The stress field being condensed, its continuity is no more ensured. Nevertheless, it has no importance since its continuous values along interfaces are given by the values of the Lagrange multipliers. Other components are calculated using equation (19) in each sub-element:

$$
\mathbb{K}=\left[\begin{array}{ccc|cc}
K_{1}^{\prime} & \mathbb{O} & \mathbb{O} & Q_{11} & \mathbb{O} \\
\mathbb{O} & K_{2}^{\prime} & \mathbb{O} & Q_{21} & Q_{22} \\
\mathbb{O} & \mathbb{O} & K_{3}^{\prime} & \mathbb{O} & Q_{32} \\
\hline{ }^{t} Q_{11} & { }^{t} Q_{21} & \mathbb{O} & \mathbb{O} & \mathbb{O} \\
\mathbb{O} & { }^{t} Q_{22} & { }^{t} Q_{32} & \mathbb{O} & \mathbb{O}
\end{array}\right]
$$

and:

$$
\mathbb{Q}=\left\{\begin{array}{c}
q^{1} \\
q^{2} \\
q^{3} \\
L^{1} \\
L^{2}
\end{array}\right\} \quad \mathbb{F}=\left\{\begin{array}{c}
f_{u^{1}}^{\prime} \\
f_{u^{2}}^{\prime} \\
f_{u^{3}}^{\prime} \\
\mathbb{O} \\
\mathbb{O}
\end{array}\right\}
$$

and:

$$
\boldsymbol{K}_{i}^{\prime}=+\boldsymbol{B}^{i} \boldsymbol{A}^{\boldsymbol{i}^{-1} t} \boldsymbol{B}^{i}
$$


The obtained system has the same number of unknowns as element 1 , but is symmetrical, which permits applications to bigger structures. Nevertheless, as for mixed systems, this formulation leads to a matrix $\mathbb{K}$ which is not definite-positive.

\section{Assessment}

In this section, we put emphasis on the behaviour of the defined elements, in order to ensure their validity.

\subsection{Eigenvalues}

The hybrid sandwich elements 1 and 3 have, at the same time, variables in displacements and Lagrange multipliers identified as stresses.

The part of their stiffness matrix related to displacements only is always definite-positive (when eigenmodes have been removed), but the global stiffness matrix is not.

To precise the phenomenon, we can say that using Lagrange multipliers to bond the sub-elements is equivalent to have 3 differents elements (not bonded) and multipliers, so that:

- for each sub-element, we have 3 zero eigenvalues in 2D (6 in 3D) corresponding to the rigid body motions, i.e. we have 9 zero eigenvalues in $2 \mathrm{D}$ and 18 in $3 \mathrm{D}$.

- for each interface, we have 2 negatives eigenvalues in 2D (3 in 3D) for each node corresponding to a Lagrange multiplier, i.e. we have 12 negative eigenvalues in $2 \mathrm{D}$ and 32 in $3 \mathrm{D}$.

- the remaining eigenvalues are positive: i.e. 31 positive eigenvalues in $2 \mathrm{D}$ and 101 in $3 \mathrm{D}$.

\subsection{Patch-Test}

We now verify what gives the patch test defined in figure 3 .

We choose to impose displacements at external nodes according to the polynomial:

$$
P(x, y)=1+x+y
$$


and we must control that the displacement values obtained with the finite element method at internal nodes correspond to the values of this polynomial at these internal nodes.

We can notice that the difference between the theoretical value of $P(x, y)$ at internal nodes and the value obtained with the finite element method is equal to $0.78 \%$ for displacements in $x$ direction and to $0.80 \%$ for displacements in $y$ direction (to be compared with 8node quadrilateral elements Plane 82 of ANSYS, where this difference is equal to $0.73 \%$ for displacements in $x$ direction and to $0.78 \%$ for displacements in $y$ direction).

\section{Examples}

In this part, we propose to solve some examples with the developed elements.

\subsection{Sandwich beam}

The 2D elements will be used to compute the simply supported sandwich beam under uniform pressure given in figure 4 .

The total length of the beam is $L=24 \mathrm{~mm}$, its total heigth $H=2 \mathrm{~mm}$, the tickness of the core is $H_{c}=1.6 \mathrm{~mm}$ ( $80 \%$ of the total height). The uniform pressure is $q=1 \mathrm{~N} / \mathrm{mm}^{2}$ (the width is taken equal to $1 \mathrm{~mm}$ ). Skins are made of aluminium with characteristics $E_{s}=70000 \mathrm{MPa}$ and $\nu_{s}=0.34$ and the core is made of epoxy $E_{c}=3400 \mathrm{Mpa}$ and $\nu_{c}=0.34$. The beam is simply supported at its ends (boundary condition: $U_{2}=0$ ), and using the symmetry of the problem, only a half beam is modelled, applying the symmetry condition $U_{1}=0$ along the mid section $\left(x_{1}=L / 2\right)$.

For this example, our reference solution will be given by Lerooy ${ }^{9}$.

The mesh is done in 2D. It is regular: 1 element through the thickness of each skin, 2 through the thickness of the core, and ncuts regular cuts in the longitudinal direction $x_{1}$.

For ncuts $=20$, this mesh corresponds to:

- 578 DOF for the displacement model;

- 1445 DOF for the model based on Reissner's functionnal and for model 2; 
- 906 DOF for the sandwich elements 1 and 3.

Our first interest is for the convergence of the central deflection of the beam. The results are presented in figures 5 and 6 .

Some remarks can be done:

- figure 5 gives the convergence as a function of the number of longitudinal cuts of the half beam, ncuts. In all cases, bi-quadratic shape functions are used in each layers for all kinds of unknowns. The convergence rate is the same for all presented methods (displacements, Reissner and Elements 1, 2 and 3).

- figure 6 gives the same curve, but as a function of the number of DOF.

The curve corresponding to Reissner's elements, where all displacements and stresses appear as nodal unknowns, is shifted to the right (the convergence rate is worst in terms of the number of DOF).

The curve corresponding to hybrid sandwich elements 1 and 3, where only few DOF corresponding to Lagrange multipliers are added at interfaces, is shifted to the right too, but the convergence rate remains better than for Reissner's elements.

Hence, we ascentain that the convergence rate, in terms of number of DOF, is fully acceptable for developed hybrid sandwich elements.

We have to keep in mind, that hybrid sandwich elements have been developed in order to obtain the values of $\boldsymbol{T}$ at interfaces. It is therefore indispensable to look at the stress distribution through the thickness of the beam. These results are presented in figures 7 and 8 for the section corresponding to the quarter of the beam $\left(x_{1}=L / 4\right)$.

The following conclusions can be drawn:

- figure 7 gives the distribution of transversal shear stress $\sigma_{12}$ through the thickness. This component must be continuous at interfaces: the solution obtained with elements based on displacements is not shown because this method is not able to leads to the continuity.

Hybrid sandwich elements yield correct values for this component as well as Reissner's elements, and especially at interfaces. Its distribution through the thickness is continuous. 
- figure 8 gives the distribution of the normal stress $\sigma_{11}$ through the thickness. This component must be discontinuous in this case.

Reissner's elements and Pian and Tong elements (element 2) lead to the continuity of the stress component at interface, which is excessive. Hybrid sandwich elements 1 and 3 do not yield the continuity of this component, since no Lagrange multiplier is associated with it. We compute this discontinuous component in the same manner as the displacement elements, i.e. element by element.

After this first example, we can already say the hybrid sandwich elements 1 and 3 only yields required continuity. Displacements elements show a weakness in terms of stress continuity (since no component is continuous), and Reissner's elements an excessive continuity (since $\sigma_{11}$ is always continuous).

\subsection{Sandwich plate}

The previous example is then extended to the $3 \mathrm{D}$ case, i.e. to the case of the simply supported square plate under uniform pressure, as depicted in figure 9.

The total length of a side is $L=24 \mathrm{~mm}$, the total heigth $H=$ $2 \mathrm{~mm}$, the tickness of the core is $H_{c}=1.6 \mathrm{~mm}$ ( $80 \%$ of the total height). The uniform pressure is $q=1 \mathrm{~N} / \mathrm{mm}^{2}$. Skins are made of aluminium with characteristics $E_{s}=70000 \mathrm{MPa}$ and $\nu_{s}=0.34$ and the core is made of epoxy $E_{c}=3400 \mathrm{Mpa}$ and $\nu_{c}=0.34$. The square plate is simply supported at its boundary (boundary condition on the bottom face: $U_{3}=0$ at $x_{1}=0, x_{1}=L, x_{2}=0$ and $\left.x_{2}=L\right)$, and using the symmetry of the problem, only a quarter of th plate is modelled, appliying the symmetry condition $U_{1}=0$ along $x_{1}=L / 2$ and $U_{2}=0$ along $x_{2}=L / 2$.

The reference solution is the three-dimensional solution of elasticity given by Pagano ${ }^{10}$.

We saw in last example that ncuts $=20$ is fully sufficient to obtain convergence. We use a regular mesh, with 4 elements through the entire thickness (as before) and 20 cuts in $x_{1}$ and $x_{2}$ directions. This mesh includes: 
- 16338 DOF for the displacement model;

- 40845 DOF for Reissner and Pian and Tong models;

- 26586 DOF for sandwich models.

Keeping the previous example in mind, we limit our study to the distribution of components $\sigma_{13}$ and $\sigma_{33}$ of stresses through the thickness of the plate. The results are presented and compared in figures 10 and 11 .

\subsection{Adhesive joint}

In order to illustrate that the present hybrid sandwich elements can be applied to similar problems, let us consider the adhesive joint shown in figure 12. The mesh is given in figure 13 .

The interface between the glue and the substrate is done by the Lagrange multipliers. The rest of the elements constituting the substrate are 8-nodes quadrilateral displacement elements. The mesh is not regular, but the ratio of the length over the width of each element varie from 1 to 20. Along the exterior surface, located at $x=0.5$, this ratio is equal to 4.28 in the glue (grayed part), 10 over quite all the substrate and 5 at the really end of the substrate. This mesh has 1004 DOF (966 in displacements and 38 corresponding to Lagrange multipliers).

Our solutions are compared with those of Gilibert ${ }^{11}$.

In this this case, skins are very thick and the core is thin. The structure is obviously not a typical sandwich, but developed elements are able to compute this type of structure too.

Only the distributions of stress components $\sigma_{12}$ and $\sigma_{22}$, which must be continuous along the interface between the glue and the skin are presented and compared. Solutions are reported in figures 14 and 15.

The present hybrid sandwich elements are in good agreement with the reference solution. 


\section{Conclusion}

Lagrange multipliers permit to model in a simple way problems related to interfaces, without changing the formulation of a any of the sub-domains. They also allow to choose, for each layer, the most appropriated formulation for a given problem.

In this study, we presented the formulation of hybrid sandwich elements using Lagrange multipliers (elements 1 and 3) to obtain interlaminar stresses at interfaces verifying the force equilibrium state. These elements have been successfully assessed, not only for sandwich beams, but also for sandwich plates and adhesive joints.

Finally, we can remark that, after having computed stresses at interfaces, it is possible to see if they fulfill a chosen rupture criterion (which is generally expressed in terms of stresses). It is then possible to suppress the Lagrange multipliers on a part of the model: it corresponds to the simulation of the delamination between layers, without having to change anything else in the formulation. This possibility, of introducing a disbond, can be particularly interesting in the case of local buckling in sandwiches, which leads to local disbonds between layers.

\section{References}

[1] R. Teti and G. Caprino, 'Mechanical behavior of structural sandwiches', Sandwich Construction 1, K.-A. Olsonn and R.P. Reichard, editors, Chameleon Press LTD., London, 53-67, (1989).

[2] D. Zenkert, An introduction to Sandwich Construction, Chameleon Press LTD., London, (1995).

[3] H.G. Allen, Analysis and Design of Structural Sandwich Panels, Pergamon Press, Oxford, (1969).

[4] H.G. Allen, 'Sandwich Construction Today and Tomorrow', Sandwich Constructions 1, K.-A. Olsonn and R.P. Reichard, editors. Chameleon Press LTD., London, 3-23, (1989).

[5] N.J. Hoff, Sandwich Construction, John Wiley \& Sons, New.York, (1966).

[6] P. Couvrat, Le collage structural moderne. Théorie $\&$ pratique., TEC \& DOC - Lavoisier, Paris, (1992). 
[7] S. Aivaz-Zadeh Mokari, Éléments finis d'interface. Application aux assemblages collés et structures stratifiées. PhD thesis, U.T.C., 1984.

[8] K. Washizu. Variational methods in elasticity and plasticity. Pergamon Press, (1982).

[9] J.-F. Lerooy. Calcul des contraintes de cisaillement transversales dans les structures modérément épaisses. $\mathrm{PhD}$ thesis, Institut National Polytechnique de Lorraine, (1983).

[10] N.J. Pagano. 'Exact solutions for rectangular bidirectional composites and sandwich plates', J. Composite Materials, 4:20-34, (1970).

[11] Y. Gilibert. Contribution à l'étude de l'adhésivité de matériaux collés par l'intermédiaire de résines époxydiques. $\mathrm{PhD}$ thesis, Reims, (1978). 


\section{List of Figures}

1 Sub-domains . . . . . . . . . . . 20

2 Hybrid sandwich finite element . . . . . . . . . 21

3 Patch test . . . . . . . . . . . . . . 22

4 Sandwich beam . . . . . . . . . . . . 23

5 Convergence of displacements as a function of ncuts . 24

6 Convergence of displacements as a function of number of DOF . . . . . . . . . . . . . 25

7 Transverse shear stress distribution through the thickness 26

8 Normal stress $\sigma_{11}$ distribution through the thickness . 27

9 Sandwich plate . . . . . . . . . . . . 28

$10 \sigma_{23}$ distribution through the thickness . . . . . . . 29

$11 \sigma_{33}$ distribution through the thickness . . . . . . . 30

12 Adhesive joint . . . . . . . . . . . . 31

13 Mesh of a quarter of the joint . . . . . . . . . . . . 32

14 Transverse shear stress $\sigma_{12}$ along interface . . . . . . 33

15 Normal stress $\sigma_{22}$ along interface . . . . . . . . 34 


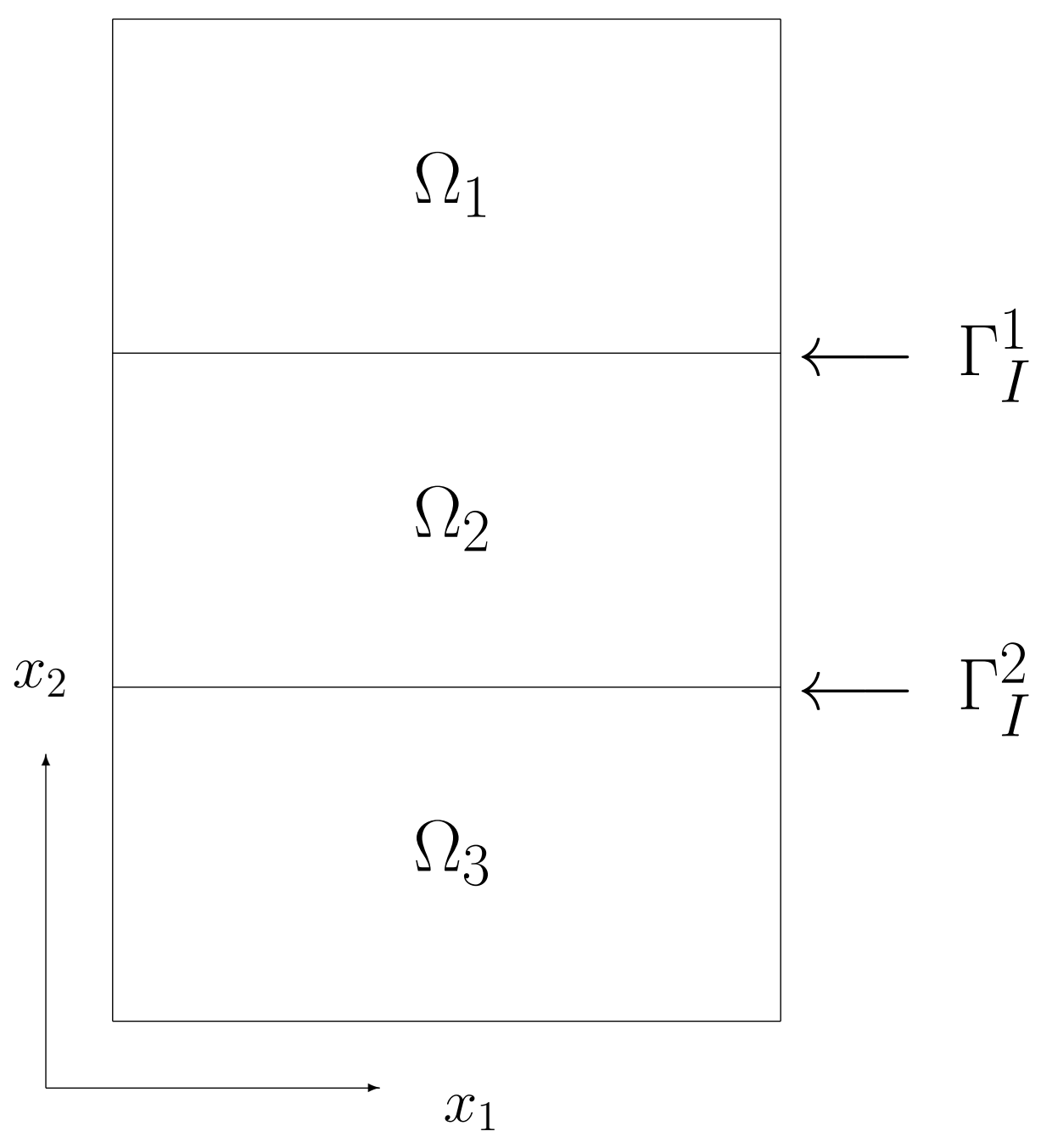

Figure 1: Sub-domains 


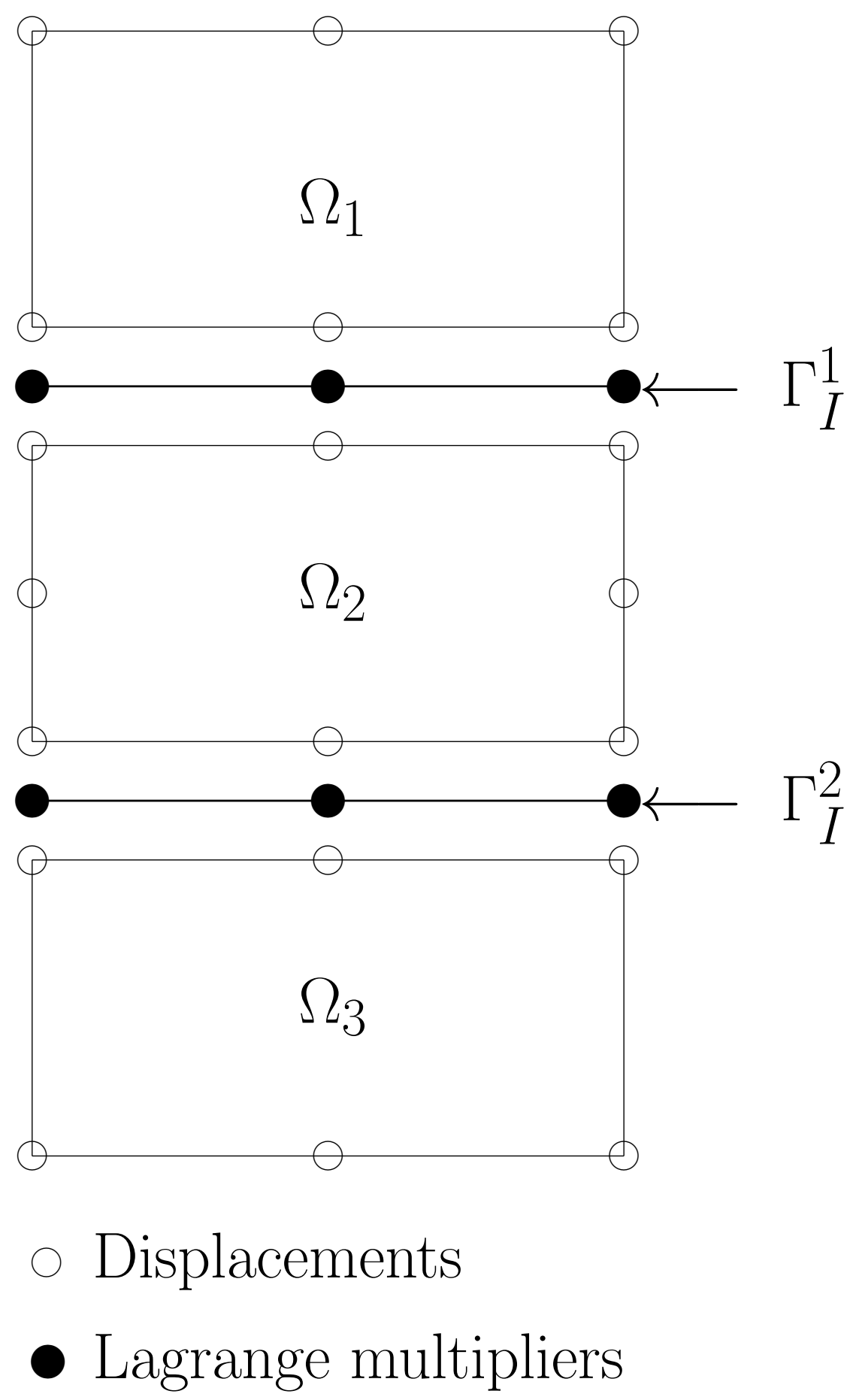

Figure 2: Hybrid sandwich finite element 


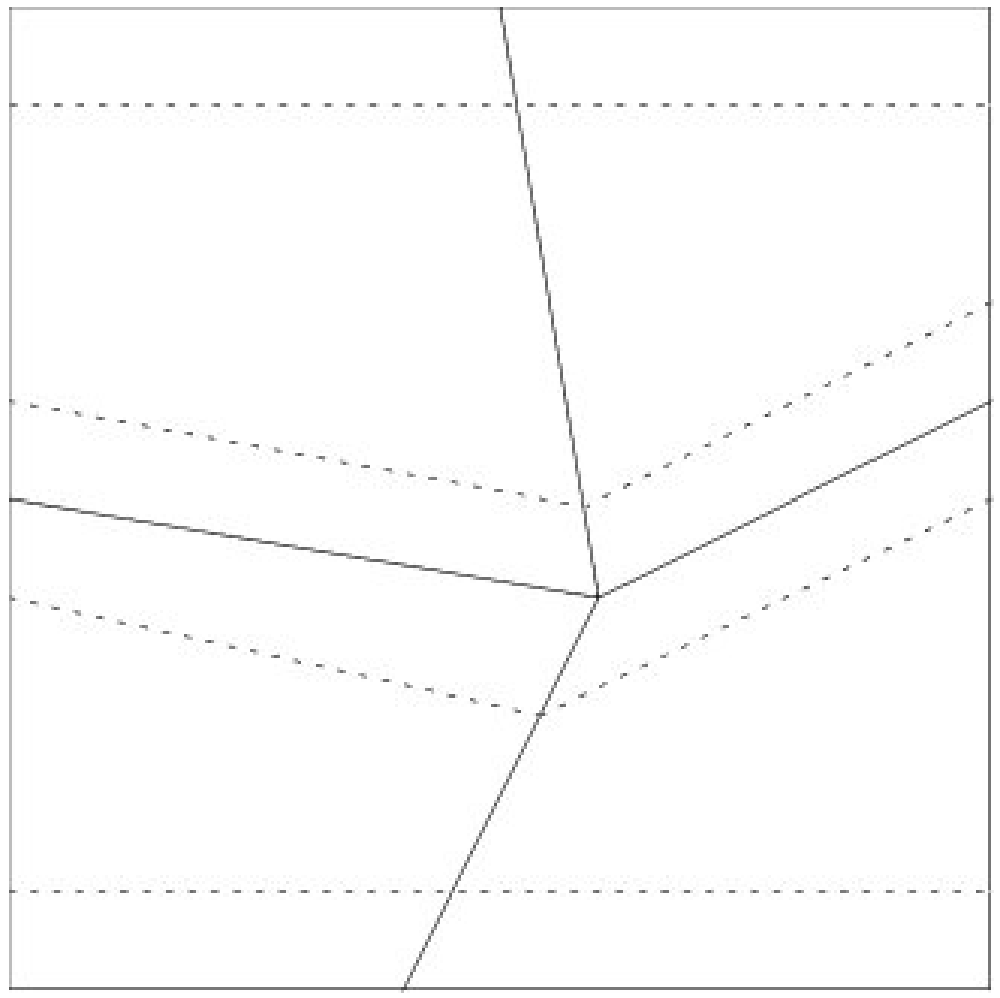

Figure 3: Patch test 


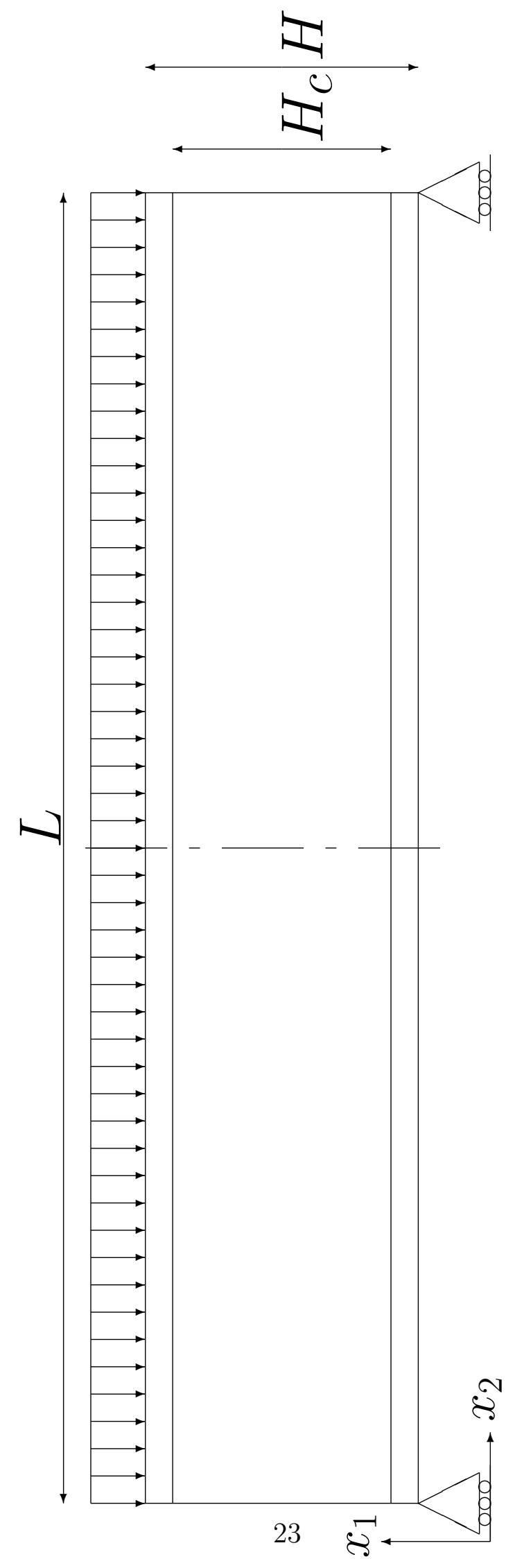

Figure 4: Sandwich beam 


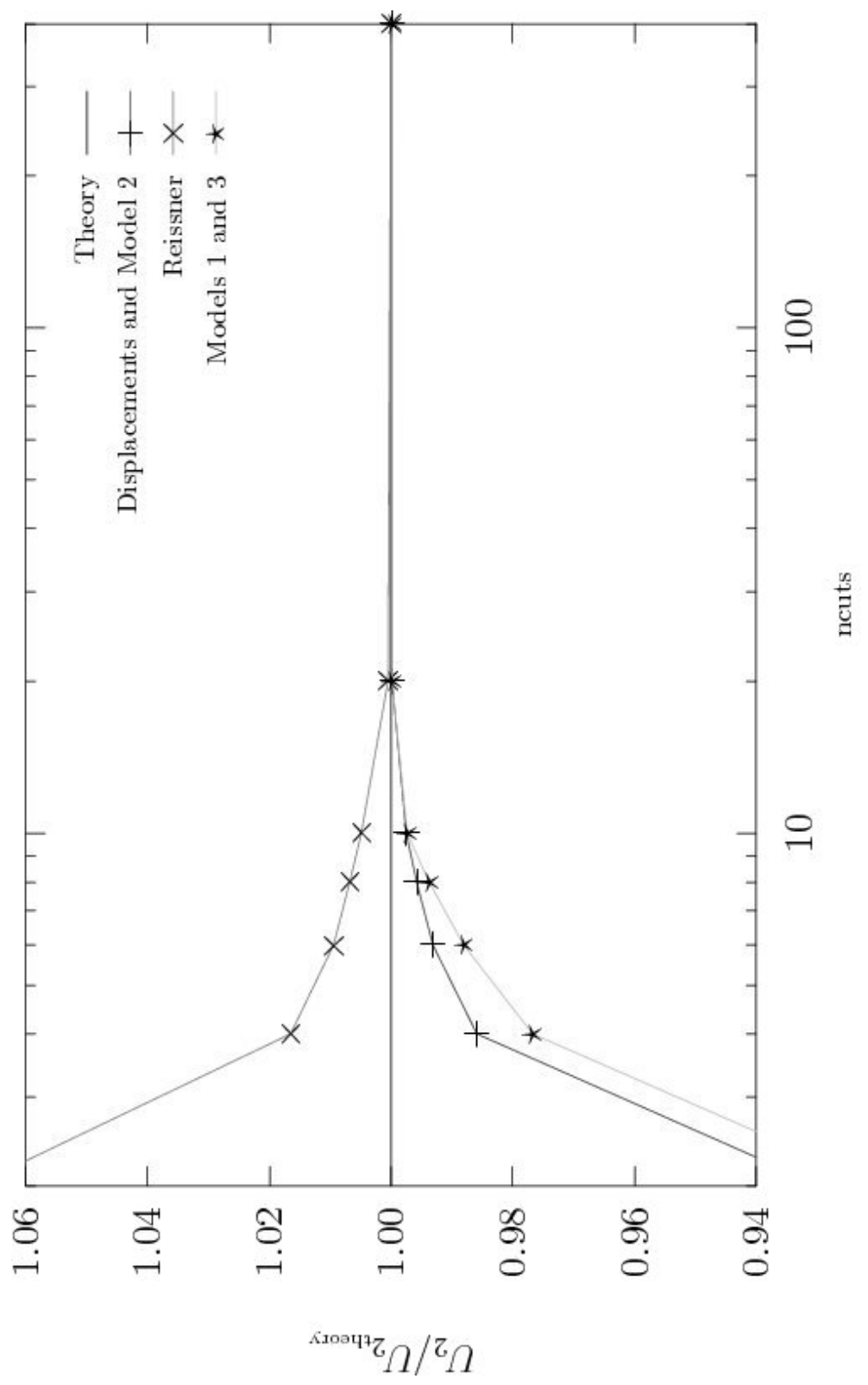

Figure 5: Convergence of displacements as a function of ncuts 


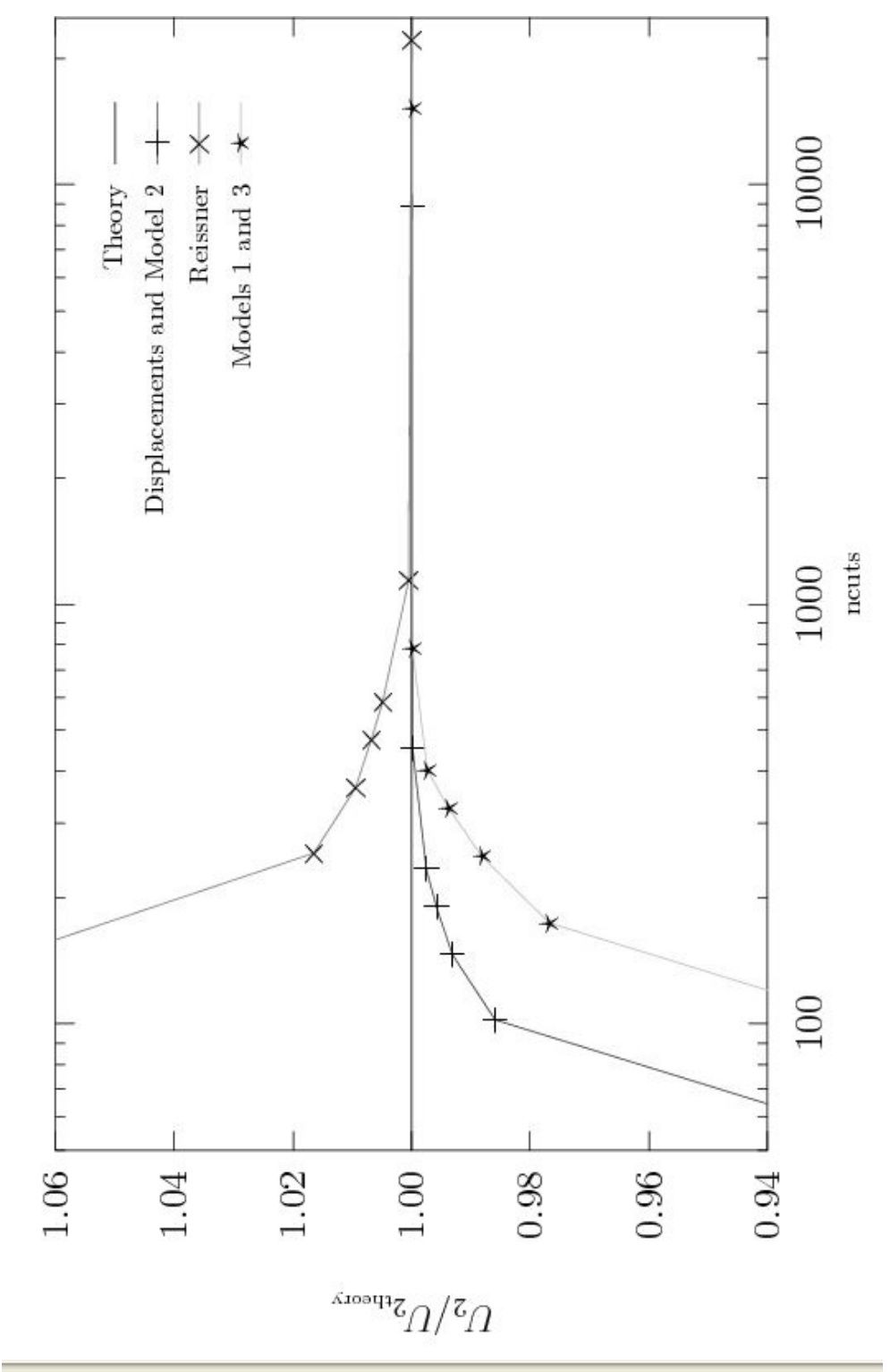

Figure 6: Convergence of displacements as a function of number of DOF 


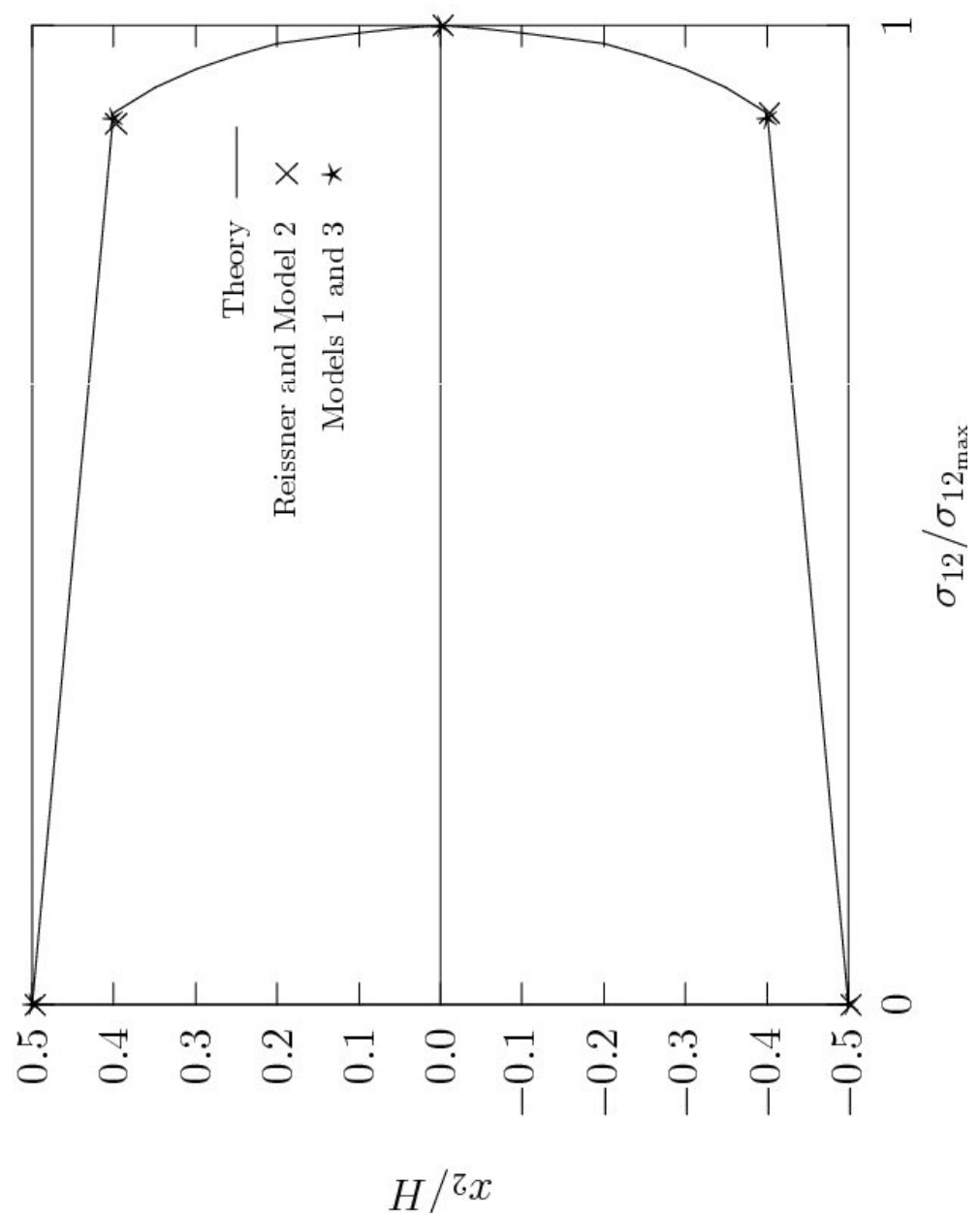

Figure 7: Transverse shear stress distribution through the thickness 


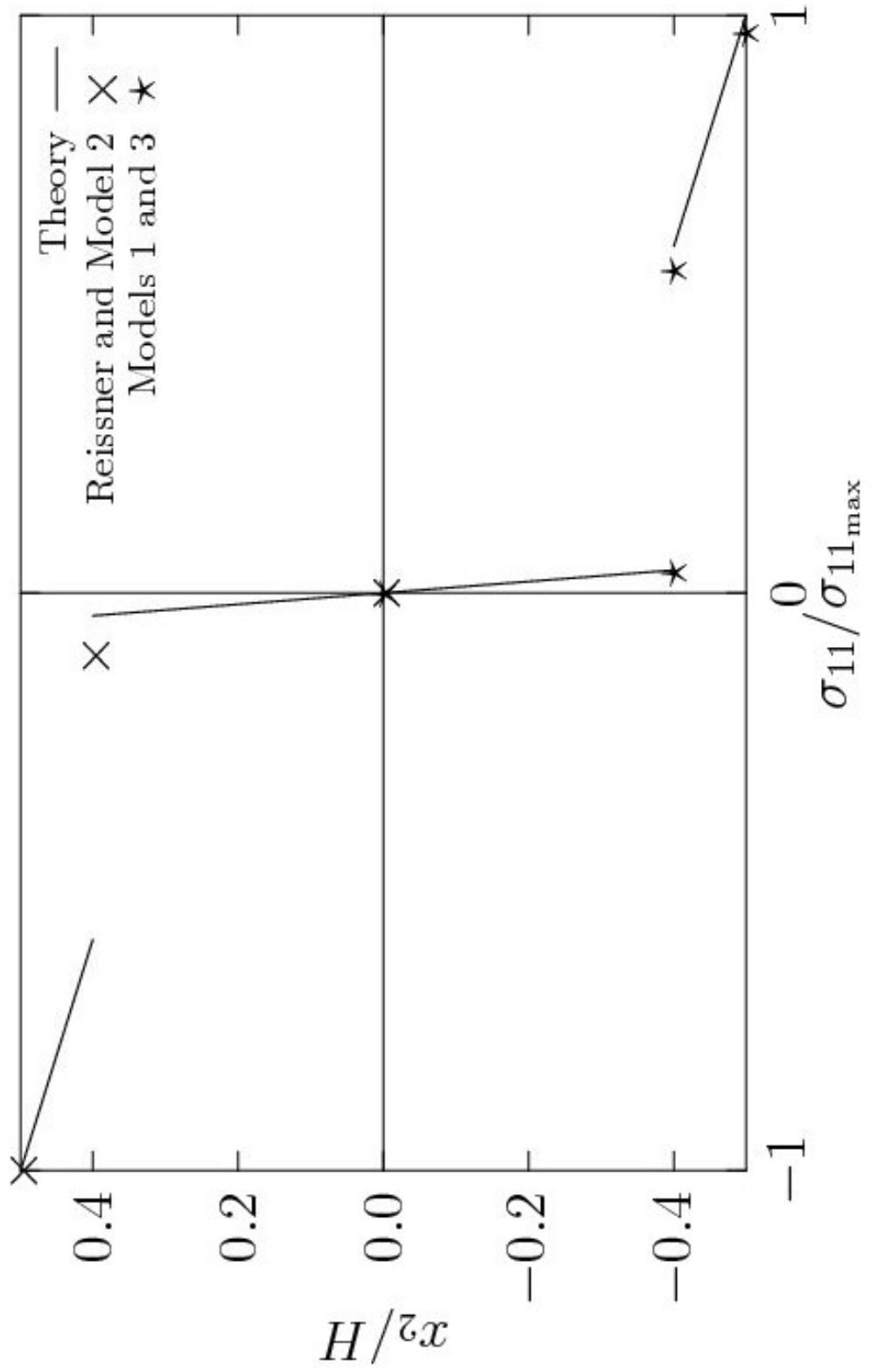

Figure 8: Normal stress $\sigma_{11}$ distribution through the thickness 

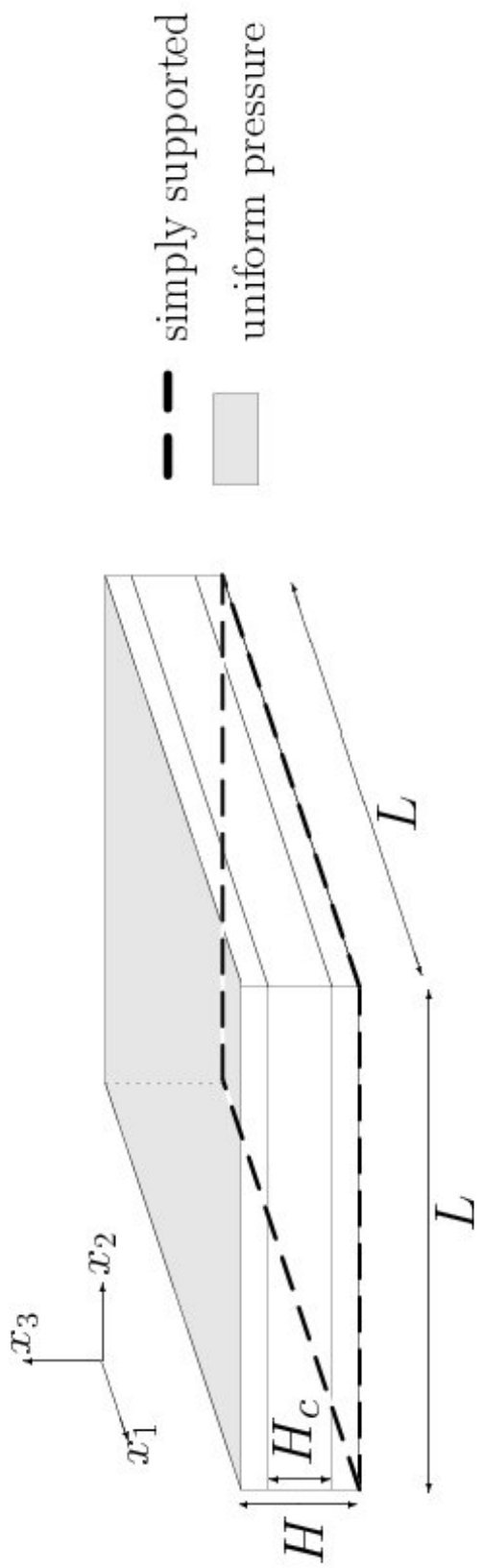

Figure 9: Sandwich plate 


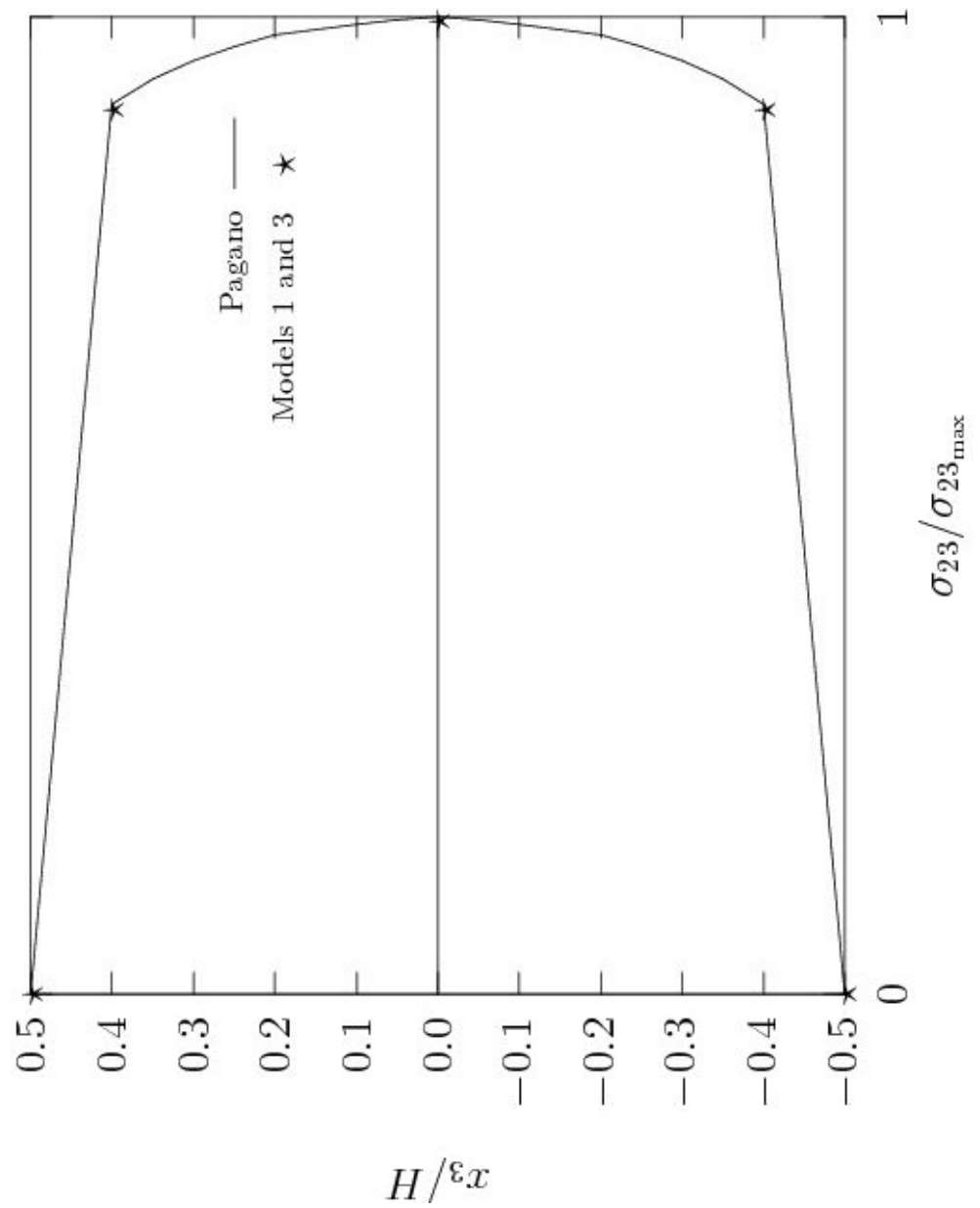

Figure 10: $\sigma_{23}$ distribution through the thickness 


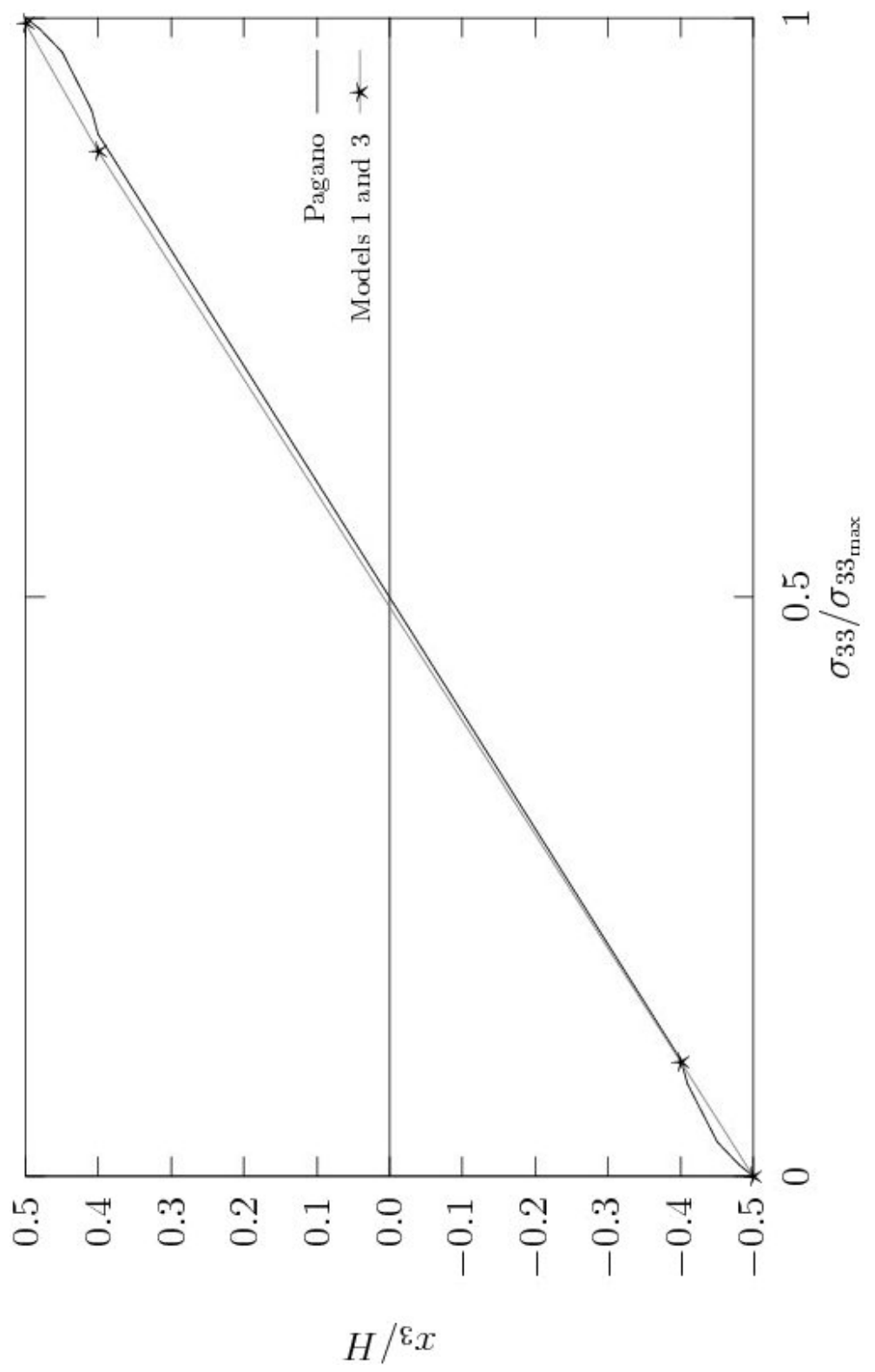

Figure 11: $\sigma_{33}$ distribution through the thickness 


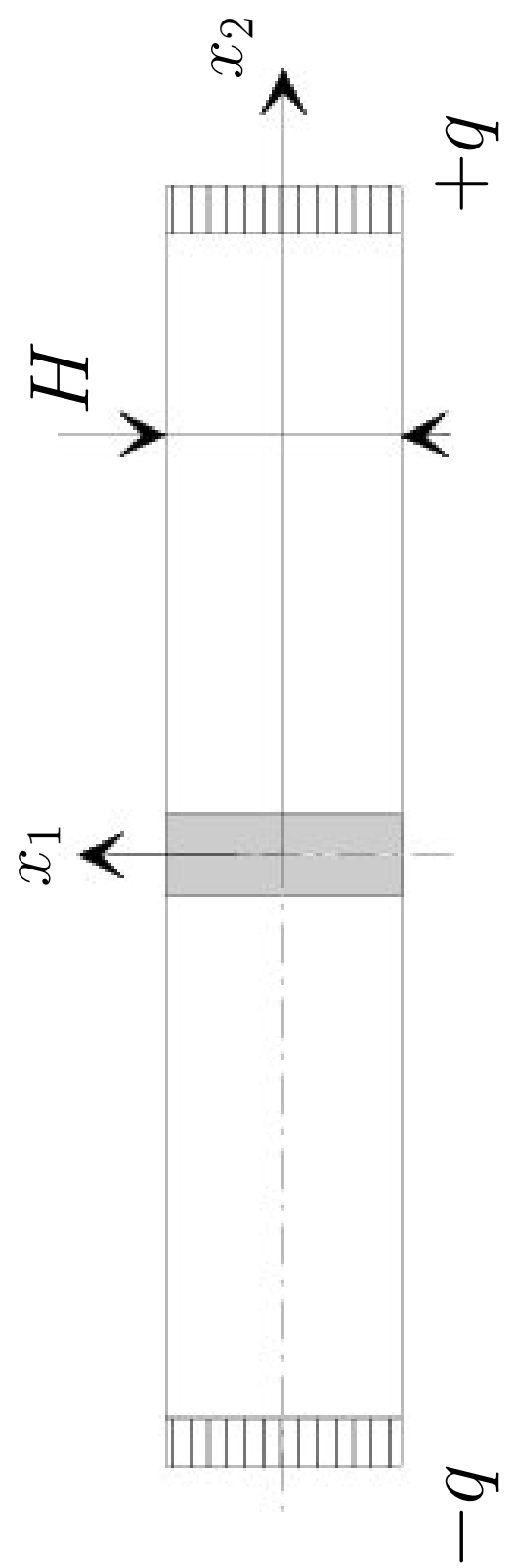

Figure 12: Adhesive joint 


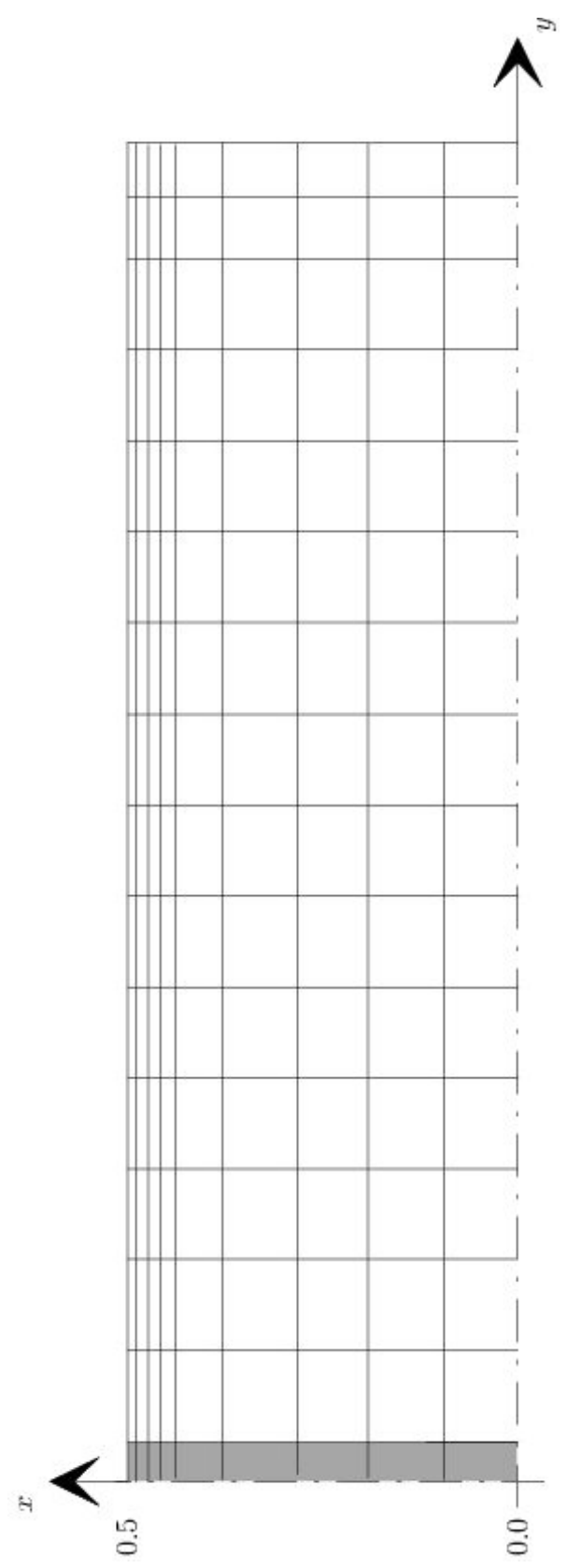

Figure 13: Mesh of a quarter of the joint 


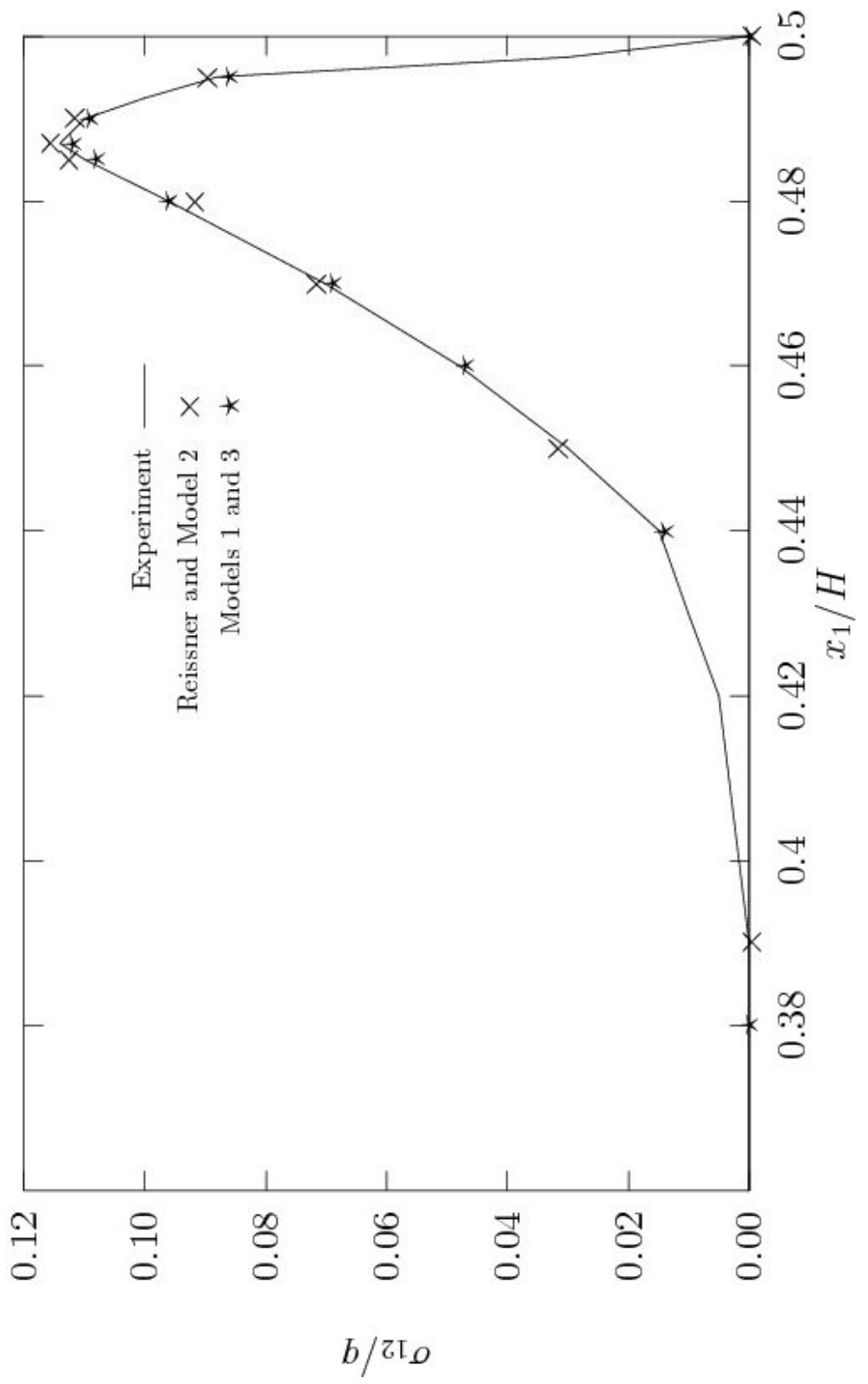

Figure 14: Transverse shear stress $\sigma_{12}$ along interface 


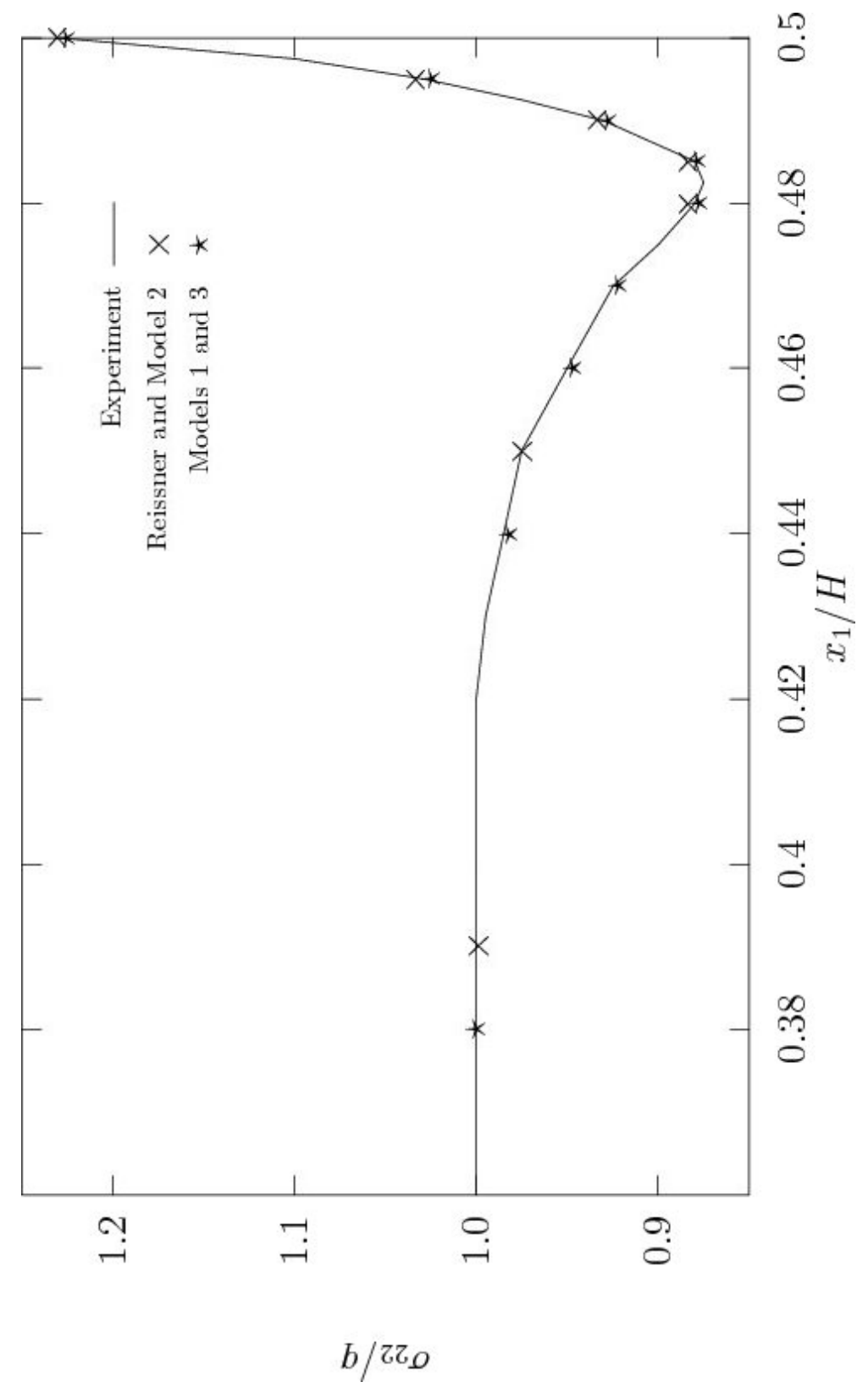

Figure 15: Normal stress $\sigma_{22}$ along interface 